\title{
Erratum to: Proprotein convertase subtilisin/kexin type 9 (PCSK9) and metabolic syndrome: insights on insulin resistance, inflammation, and atherogenic dyslipidemia
}

\author{
Nicola Ferri $^{1} \cdot$ Massimiliano Ruscica $^{2}$
}

Published online: 5 October 2016

(C) Springer Science+Business Media New York 2016

\section{Erratum to: Endocrine}

DOI 10.1007/s12020-016-0939-0

In "Glucagon and insulin" section of the original publication, it was incorrectly described as "glucose showed a strong and rapid suppression of both LDLR and PCSK9 proteins", instead of "glucagon showed a suppression of PCSK9 mRNA and protein levels". It should be read as:

"Such observation was corroborated by a study reporting how rat administered with glucagon showed a suppression of PCSK9 mRNA and protein levels, as well as a $25 \%$ reduction in SREBP-2 mRNA expression [50]."

The online version of the original article can be found under doi: 10.1007/s12020-016-0939-0.

Massimiliano Ruscica

massimiliano.ruscica@unimi.it

Nicola Ferri

nicola.ferri@unipd.it

1 Dipartimento di Scienze del Farmaco, Università degli Studi di Padova, Largo Meneghetti 2, 35131 Padua, Italy

2 Dipartimento di Scienze Farmacologiche e Biomolecolari, Università degli Studi di Milano, Via Balzaretti 9, 20133 Milan, Italy 